\title{
Exploiting Flexibility in Coupled Electricity and Natural Gas Markets: A Price-Based
} Approach

Ordoudis, Christos; Delikaraoglou, Stefanos; Pinson, Pierre; Kazempour, Jalal

\section{Published in:}

Proceedings of IEEE PES PowerTech Conference 2017

Link to article, DOI:

10.1109/PTC.2017.7981047

Publication date:

2017

Document Version

Peer reviewed version

Link back to DTU Orbit

Citation (APA):

Ordoudis, C., Delikaraoglou, S., Pinson, P., \& Kazempour, J. (2017). Exploiting Flexibility in Coupled Electricity and Natural Gas Markets: A Price-Based Approach. In Proceedings of IEEE PES PowerTech Conference 2017 IEEE. https://doi.org/10.1109/PTC.2017.7981047

\section{General rights}

Copyright and moral rights for the publications made accessible in the public portal are retained by the authors and/or other copyright owners and it is a condition of accessing publications that users recognise and abide by the legal requirements associated with these rights.

- Users may download and print one copy of any publication from the public portal for the purpose of private study or research.

- You may not further distribute the material or use it for any profit-making activity or commercial gain

- You may freely distribute the URL identifying the publication in the public portal 


\title{
Exploiting Flexibility in Coupled Electricity and Natural Gas Markets: A Price-Based Approach
}

\author{
Christos Ordoudis, Stefanos Delikaraoglou, Pierre Pinson and Jalal Kazempour \\ Department of Electrical Engineering \\ Technical University of Denmark \\ Kgs. Lyngby, Denmark \\ \{chror, stde, ppin, seykaz\}@dtu.dk
}

\begin{abstract}
Natural gas-fired power plants (NGFPPs) are considered a highly flexible component of the energy system and can facilitate the large-scale integration of intermittent renewable generation. Therefore, it is necessary to improve the coordination between electric power and natural gas systems. Considering a market-based coupling of these systems, we introduce a decision support tool that increases market efficiency in the current setup where day-ahead and balancing markets are cleared sequentially. The proposed approach relies on the optimal adjustment of natural gas price to modify the scheduling of power plants and reveals the necessary flexibility to handle stochastic renewable production. An essential property of this price-based approach is that it guarantees no financial imbalance (deficit or surplus) for the system operator at the day-ahead stage. Our analysis shows that the proposed mechanism reduces the expected system cost and efficiently accommodates high shares of renewables.
\end{abstract}

Index Terms-Bilevel programming, electricity markets, natural gas markets, stochastic programming.

\section{NOMENCLATURE}

\section{Sets}

I Set of dispatchable power production units $i$.

$I_{c} \quad$ Set of thermal power plants $i_{c}\left(I_{c} \subset I\right)$.

$I_{g} \quad$ Set of natural gas-fired power plants $i_{g}\left(I_{g} \subset I\right)$.

$J \quad$ Set of wind power units $j$.

$K \quad$ Set of natural gas production units $k$

$L \quad$ Set of natural gas pipelines $l$.

$A_{l}^{i_{g}} \quad$ Set of natural gas-fired power plants $i_{g}$ at pipeline $l$.

$\Omega \quad$ Set of wind power scenarios $\omega$.

$T \quad$ Set of time periods $t$.

Variables

$p_{i, t}, w_{j, t}$ Day-ahead dispatch of units $i$ and $j$ in period $t$ [MW].

$p_{i, \omega, t}^{+l-} \quad \mathrm{Up} /$ down regulation provided by unit $i$ in scenario $\omega$, period $t$ [MW].

$w_{j, \omega, t}^{\mathrm{sp}} \quad$ Wind power spilled by unit $j$ in scenario $\omega$, period $t$ [MW].

$l_{\omega, t}^{\mathrm{sh}, \mathrm{E}}, l_{\omega, t}^{\mathrm{sh}, \mathrm{G}}$ Electricity and natural gas load shedding in scenario $\omega$, period $t\left[\mathrm{MW}, \mathrm{kNm}^{3} / \mathrm{h}\right]$.

$g_{k, t} \quad$ Day-ahead dispatch of unit $k$ in period $t\left[\mathrm{kNm}^{3} / \mathrm{h}\right]$.

$g_{k, \omega, t}^{+/-} \quad$ Up/down regulation provided by unit $k$ in scenario $\omega$, period $t\left[\mathrm{kNm}^{3} / \mathrm{h}\right]$.

$x_{t} \quad$ Natural gas price adjustment $\left[\$ / \mathrm{kNm}^{3}\right]$.

$\lambda, \mu \quad$ Dual variables of equality and inequality constraints.

\section{Parameters}

$D_{t}^{\mathrm{E}} \quad$ Electricity demand in period $t$ [MW].

$D_{t}^{\mathrm{G}} \quad$ Natural gas demand in period $t\left[\mathrm{kNm}^{3} / \mathrm{h}\right]$.

$C_{i} \quad$ Day-ahead offer price of unit $i$ [\$/MWh].

$C_{i}^{+/-} \quad$ Up/down regulation offer price of unit $i$ [\$/MWh].

$C^{\text {sh,E }} \quad$ Cost of electricity load shedding [\$/MWh].

$C^{\mathrm{sp}} \quad$ Cost of wind power spillage [\$/MWh].
$C_{k} \quad$ Day-ahead offer price of unit $k\left[\$ / \mathrm{kNm}^{3}\right]$.

$C_{k}^{+/-} \quad$ Up/down regulation offer price of unit $k\left[\$ / \mathrm{kNm}^{3}\right]$.

$C^{\mathrm{sh}, \mathrm{G}} \quad$ Cost of natural gas load shedding $\left[\$ / \mathrm{kNm}^{3}\right]$.

$P_{i}^{\max } \quad$ Capacity of dispatchable unit $i$ [MW].

$P_{i}^{+/-} \quad$ Up/down reserve offer by unit $i$ [MW].

$\phi_{i_{g}} \quad$ Power conversion factor of unit $i_{g}\left[\mathrm{kNm}^{3} / \mathrm{MWh}\right]$.

$W_{j, \omega, t} \quad$ Wind power realization in scenario $\omega$, period $t$ [MW].

$\widehat{W}_{j, t} \quad$ Expected wind power by unit $j$ in period $t$ [MW].

$\bar{W}_{j} \quad$ Capacity of wind power unit $j$ [MW].

$G_{k}^{\max } \quad$ Capacity of natural gas unit $k\left[\mathrm{kNm}^{3} / \mathrm{h}\right]$.

$G_{k}^{+/-} \quad$ Up/down reserve offer by unit $k\left[\mathrm{kNm}^{3} / \mathrm{h}\right]$.

$X \quad$ Limit of natural gas price adjustment $\left[\$ / \mathrm{kNm}^{3}\right]$.

$F_{l, t}^{\mathrm{M}} \quad$ Capacity of natural gas pipeline $l$ in period $t\left[\mathrm{kNm}^{3} / \mathrm{h}\right]$.

$F_{l}^{\AA} \quad$ Daily contract limit of natural gas pipeline $l\left[\mathrm{kNm}^{3}\right]$.

\section{INTRODUCTION}

In recent years, renewable energy makes up a high share of the total electricity production and is expected to increase further in the future. In view of accomplishing a transition to a green energy system, natural gas-fired power plants (NGFPPs) seem an ideal choice to facilitate this shift due to their operational flexibility and high efficiency, especially if the potential of using green gases (e.g., biogas) is considered.

The tighter coupling of electricity and natural gas markets can promote the integration of renewables in the energy system. Coupling these two markets is a natural way to increase the coordination between the two systems that have existing synergies mainly through the NGFPPs. Authors in [1] and [2] study different coordination setups in short-term operational models and highlight the benefits of such coupling. The effect of natural gas supply uncertainty and price variability on the scheduling of power plants is shown in [3]. Moreover, the case of Spain that builds its energy mix on the basis of combining renewables and NGFPPs is described in [4] indicating the need for a coupled operation of the two systems. Towards that goal, several technical and regulatory challenges need to be addressed, such as the alignment of electricity and natural gas market timing, the establishment of effective mechanisms to couple the operation and the increase of short-term trading in natural gas markets. Authors in [5] extensively discuss the timing between the two markets and how this could be harmonized. In addition, short-term trading of natural gas has increased compared to previous years as spot markets (e.g., Gaspoint Nordic) continuously develop and attract larger volumes for trading [6]. These changes will facilitate the 
integration of electricity and natural gas systems to flourish under high shares of renewables. To this end, we develop a market-based coupling where the market timing is concurrent and the quantities are traded in short-term markets.

Current market designs that are based on the sequential clearing of the day-ahead and balancing trading floors may result in significant balancing costs as the penetration of renewables increases. Recent literature, e.g. [7] - [9], discusses the use of stochastic programming to anticipate future balancing needs. We consider this approach as the ideal benchmark in terms of expected cost.

In this paper, we propose a setup that couples electricity and natural gas markets and bridges the efficiency gap between the sequential and stochastic models. From a physical perspective, these two systems interact through the natural gas consumption of NGFPPs, while from an economic viewpoint they are implicitly coordinated through the natural gas price offered to NGFPPs. Exploiting this economic link, we propose a stochastic bilevel model that explicitly captures the temporal coordination between the day-ahead and balancing markets, as well as it respects the existing sequential market structure. This approach is price-based and allows the system operator to optimally adjust the natural gas price offered to NGFPPs by providing proper flexibility price signals. These price signals aim at modifying the unit dispatch and revealing adequate flexibility to cope with real-time imbalances. This mechanism is designed on a cost-neutral basis to ensure that the system operator will be financially balanced at the day-ahead stage. Nevertheless, this action may lead to potential real-time deficit or surplus that can be considered a price-based incentive to promote power system flexibility, akin to the flexible capacity remuneration mechanisms that are currently under discussion in the European electricity market context [10]. Results show a sound increase of market efficiency by the proposed model, as the expected system cost significantly reduces via an improved unit dispatch that effectively handles stochastic production.

This paper is organized as follows. In Sections II-A and II-B, we present the sequential and stochastic dispatch models, respectively. We describe the proposed price-based mechanism in Section II-C. Section III demonstrates the results on a stylized case study, while Section IV concludes the paper.

\section{DISPATCH MODELS}

The models considered in this study assume that electricity and natural gas markets are coupled, i.e., the market clearing is a single optimization problem. This approach is an optimistic view of the current setup, where these markets are actually decoupled and thus loosely coordinated in terms of price, i.e., NGFPPs submit their electricity price-quantity offers based on an estimation and not the true value of the natural gas price.

\section{A. Sequential Coupled Electricity and Natural Gas Model}

The sequential dispatch model (Seq) clears independently the day-ahead and balancing markets. The optimal schedule that minimizes the day-ahead cost of the integrated system is determined by model (1) as follows,

$$
\underset{\Theta^{\mathrm{D}}}{\operatorname{Min}} \sum_{t \in T}\left(\sum_{i_{c} \in I_{c}} C_{i_{c}} p_{i_{c}, t}+\sum_{k \in K} C_{k} g_{k, t}\right)
$$

subject to

$$
\begin{aligned}
& 0 \leq p_{i, t} \leq P_{i}^{\max }: \underline{\mu}_{i, t}^{\mathrm{P}}, \bar{\mu}_{i, t}^{\mathrm{P}}, \quad \forall i, t, \\
& 0 \leq w_{j, t} \leq \widehat{W}_{j, t}: \underline{\mu}_{j, t}^{\widehat{\mathrm{W}}}, \bar{\mu}_{j, t}^{\widehat{\mathrm{W}}}, \quad \forall j, t, \\
& \sum_{i \in I} p_{i, t}+\sum_{j \in J} w_{j, t}-D_{t}^{\mathrm{E}}=0: \hat{\lambda}_{t}^{\mathrm{E}}, \quad \forall t, \\
& 0 \leq \sum_{i_{g} \in A_{l}^{I_{g}}} \phi_{i_{g}} p_{i_{g}, t} \leq F_{l, t}^{\mathrm{M}}: \underline{\mu}_{l, t}^{\mathrm{M}}, \bar{\mu}_{l, t}^{\mathrm{M}}, \quad \forall l, t, \\
& 0 \leq \sum_{t \in T} \sum_{i_{g} \in A_{l}^{I_{g}}} \phi_{i_{g}} p_{i_{g}, t} \leq F_{l}^{\mathrm{A}}: \underline{\mu}_{l}^{\mathrm{A}}, \bar{\mu}_{l}^{\mathrm{A}}, \quad \forall l, \\
& 0 \leq g_{k, t} \leq G_{k}^{\max }: \underline{\mu}_{k, t}^{\mathrm{G}}, \bar{\mu}_{k, t}^{\mathrm{G}}, \quad \forall k, t, \\
& \sum_{k \in K} g_{k, t}-D_{t}^{\mathrm{G}}-\sum_{i_{g} \in I_{g}} \phi_{i_{g}} p_{i_{g}, t}=0: \hat{\lambda}_{t}^{\mathrm{G}}, \quad \forall t,
\end{aligned}
$$

where $\Theta^{\mathrm{D}}=\left\{p_{i, t}, \forall i, t ; w_{j, t}, \forall j, t ; g_{k, t}, \forall k, t\right\}$ is the set of primal optimization variables. The total operating cost in (1a) stems from the power production cost of thermal power plants and the total natural gas production cost. The power production cost of NGFPPs is neglected as this would imply double counting it. Constraints (1b) and (1c) enforce the upper and lower limits of power production of dispatchable and wind power plants. Wind power is constrained by the expected wind generation. Equations (1d) and (1h) represent the power and natural gas balance at the day-ahead stage. The physical pipeline capacity in each hour is imposed by (1e), while (1f) limits the daily natural gas use. The natural gas production limits of each plant are determined through $(1 \mathrm{~g})$. For the sake of conciseness, we denote the regulation provided by each balancing power plant as $\Delta p_{i, \omega^{\prime}, t}=p_{i, \omega^{\prime}, t}^{+}-p_{i, \omega^{\prime}, t}^{-}$. Having the day-ahead schedule of the integrated system as a fixed parameter (denoted with superscript ' $*$ ') and for a specific realization of $W_{j, \omega^{\prime}, t}$, the real-time market clearing writes as,

$$
\begin{aligned}
& \underset{\Theta^{\mathrm{R}}}{\operatorname{Min}} \sum_{t \in T}\left(\sum_{k \in K}\left(C_{k}^{+} g_{k, \omega^{\prime}, t}^{+}-C_{k}^{-} g_{k, \omega^{\prime}, t}^{-}\right)+C^{\mathrm{sh}, \mathrm{E}} l_{\omega^{\prime}, t}^{\mathrm{sh}, \mathrm{E}}\right. \\
& \left.+\sum_{i_{c} \in I_{c}}\left(C_{i_{c}}^{+} p_{i_{c}, \omega^{\prime}, t}^{+}-C_{i_{c}}^{-} p_{i_{c}, \omega^{\prime}, t}^{-}\right)+C^{\mathrm{sh}, \mathrm{G}} l_{\omega^{\prime}, t}^{\mathrm{sh}, \mathrm{G}}+\sum_{j \in J} C^{\mathrm{sp}} w_{j, \omega^{\prime}, t}^{\mathrm{sp}}\right)
\end{aligned}
$$

subject to

$$
\begin{aligned}
& 0 \leq p_{i, \omega^{\prime}, t}^{+} \leq P_{i}^{\max }-p_{i, t}^{*}: \underline{\mu}_{i, \omega^{\prime}, t}^{\mathrm{PR}+}, \bar{\mu}_{i, \omega^{\prime}, t}^{\mathrm{PR}+}, \quad \forall i, t, \\
& 0 \leq p_{i, \omega^{\prime}, t}^{-} \leq p_{i, t}^{*}: \underline{\mu}_{i, \omega^{\prime}, t}^{\mathrm{PR}-}, \bar{\mu}_{i, \omega^{\prime}, t}^{\mathrm{PR}-}, \quad \forall i, t, \\
& 0 \leq p_{i, \omega^{\prime}, t}^{+} \leq P_{i}^{+}: \underline{\mu}_{i, \omega^{\prime}, t}^{\mathrm{P}+}, \bar{\mu}_{i, \omega^{\prime}, t}^{\mathrm{PP}}, \quad \forall i, t, \\
& 0 \leq p_{i, \omega^{\prime}, t}^{-} \leq P_{i}^{-}: \underline{\mu}_{i, \omega^{\prime}, t}^{\mathrm{P}-}, \bar{\mu}_{i, \omega^{\prime}, t}^{\mathrm{P}-}, \quad \forall i, t, \\
& 0 \leq w_{j, \omega^{\prime}, t}^{\mathrm{sp}} \leq W_{j, \omega^{\prime}, t}: \underline{\mu}_{j, \omega^{\prime}, t}^{\mathrm{sp}}, \bar{\mu}_{j, \omega^{\prime}, t}^{\mathrm{sp}}, \quad \forall j, t, \\
& 0 \leq l_{\omega^{\prime}, t}^{\mathrm{sp}, \mathrm{E}} D_{t}^{\mathrm{E}}: \underline{\mu}_{\omega^{\prime}, t}^{\mathrm{sh}, \mathrm{E}}, \bar{\mu}_{\omega^{\prime}, t}^{\mathrm{sh}}, \quad \forall t, \\
& \sum_{i \in I} \Delta p_{i, \omega^{\prime}, t}+l_{\omega^{\prime}, t}^{\mathrm{sh}, \mathrm{E}} \\
& +\sum_{j \in J}\left(W_{j, \omega^{\prime}, t}-w_{j, \omega^{\prime}, t}^{\mathrm{sp}}-w_{j, t}^{*}\right)=0: \tilde{\lambda}_{\omega^{\prime}, t}^{\mathrm{E}}, \quad \forall t,
\end{aligned}
$$




$$
\begin{aligned}
& 0 \leq \sum_{i_{g} \in A_{l}^{I g}} \phi_{i_{g}}\left(p_{i_{g}, t}^{*}+\Delta p_{i_{g}, \omega^{\prime}, t}\right) \leq F_{l, t}^{\mathrm{M}}: \underline{\mu}_{l, \omega^{\prime}, t}^{\mathrm{MR}}, \bar{\mu}_{l, \omega^{\prime}, t}^{\mathrm{MR}}, \forall l, t, \\
& 0 \leq \sum_{t \in T_{i_{g} \in A_{l}^{I g}}} \sum_{i_{g}}\left(p_{i_{g}, t}^{*}+\Delta p_{i_{g}, \omega^{\prime}, t}\right) \leq F_{l}^{\mathrm{A}}: \underline{\mu}_{l, \omega^{\prime}}^{\mathrm{AR}} \bar{\mu}_{l, \omega^{\prime}}^{\mathrm{AR}}, \forall l, \\
& 0 \leq g_{k, \omega^{\prime}, t}^{+} \leq G_{k}^{\mathrm{max}}-g_{k, t}^{*}: \underline{\mu}_{k, \omega^{\prime}, t}^{\mathrm{GR}+}, \bar{\mu}_{k, \omega^{\prime}, t}^{\mathrm{GR}+}, \quad \forall k, t, \\
& 0 \leq g_{k, \omega^{\prime}, t}^{-} \leq g_{k, t}^{*}: \underline{\mu}_{k, \omega^{\prime}, t}^{\mathrm{GR}-}, \bar{\mu}_{k, \omega^{\prime}, t}^{\mathrm{GR}-}, \quad \forall k, t, \\
& 0 \leq g_{k, \omega^{\prime}, t}^{+} \leq G_{k}^{+}: \underline{\mu}_{k, \omega^{\prime}, t}^{\mathrm{G}+}, \bar{\mu}_{k, \omega^{\prime}, t}^{\mathrm{G}+}, \quad \forall k, t, \\
& 0 \leq g_{k, \omega^{\prime}, t}^{-} \leq G_{k}^{-}: \underline{\mu}_{k, \omega^{\prime}, t}^{\mathrm{G}-}, \bar{\mu}_{k, \omega^{\prime}, t}^{\mathrm{G}-}, \quad \forall k, t, \\
& 0 \leq l_{\omega^{\prime}, t}^{\mathrm{sh}, \mathrm{G}} \leq D_{t}^{\mathrm{G}}: \underline{\mu}_{\omega^{\prime}, t}^{\mathrm{sh}, \mathrm{G}}, \bar{\mu}_{\omega^{\prime}, t}^{\mathrm{sh}, \mathrm{G}}, \quad \forall t \\
& \sum_{k \in K}\left(g_{k, \omega^{\prime}, t}^{+}-g_{k, \omega^{\prime}, t}^{-}\right)+l_{\omega^{\prime}, t}^{\mathrm{sh}, \mathrm{G}} \\
& -\sum_{i_{g} \in I_{g}} \phi_{i_{g}} \Delta p_{i_{g}, \omega^{\prime}, t}=0: \tilde{\lambda}_{\omega^{\prime}, t}^{\mathrm{G}}, \forall t,
\end{aligned}
$$

where $\Theta^{\mathrm{R}}=\left\{p_{i, \omega^{\prime}, t}^{+}, p_{i, \omega^{\prime}, t}^{-}, \forall i, t ; l_{\omega^{\prime}, t}^{\mathrm{sh}, \mathrm{E}}, l_{\omega^{\prime}, t}^{\mathrm{sh}, \mathrm{G}}, \forall t ; w_{j, \omega^{\prime}, t}^{\mathrm{sp}}, \forall j, t\right.$; $\left.g_{k, \omega^{\prime}, t}^{+}, g_{k, \omega^{\prime}, t}^{-}, \forall k, t\right\}$ is the set of primal optimization variables. The aim of model (2) is to minimize the balancing cost of re-dispatch actions. Constraints (2b) and (2c) determine the bounds of power regulation taking into account the dayahead schedule and the capacity of the power plant, while (2d) and (2e) enforce the limits of up- and down-regulation. Wind spillage is restricted by the actual wind power realization and load shedding by electricity demand through (2f) and $(2 \mathrm{~g})$, respectively. Equation (2h) represents the power balance in real-time operation. The real-time physical pipeline capacity is enforced by (2i), while (2j) imposes the daily natural gas volume limit. Constraints (2k) and (2l) set the bounds of natural gas regulation given the capacity and day-ahead schedule of each plant. Up- and down-regulation levels of natural gas are limited by $(2 \mathrm{~m})$ and $(2 \mathrm{n})$, while load shedding is limited by the natural gas demand in (2o). Constraint (2p) enforces real-time natural gas balancing. In all models, the dual variables of each constraint are indicated after a colon. The dual variables of equality constraints are of particular interest since they reflect the market price for electricity and natural gas. The expected balancing cost over a scenario set $\Omega$ is given as the sum of the balancing cost for each scenario $\omega$ weighed by its probability of occurrence $\pi_{\omega}$.

\section{B. Stochastic Coupled Electricity and Natural Gas Model}

The stochastic dispatch model (Stoch) optimizes jointly the day-ahead and balancing stages of the integrated electric power and natural gas systems. The problem is formulated as a twostage stochastic program aiming to minimize the total expected cost and writes as follows,

$$
\begin{aligned}
& \underset{\Theta^{\mathrm{SC}}}{\operatorname{Min}} \sum_{t \in T}\left[\sum_{i_{c} \in I_{c}} C_{i_{c}} p_{i_{c}, t}+\sum_{k \in K} C_{k} g_{k, t}+\sum_{\omega \in \Omega} \pi_{\omega}(\right. \\
& \sum_{k \in K}\left(C_{k}^{+} g_{k, \omega, t}^{+}-C_{k}^{-} g_{k, \omega, t}^{-}\right)+\sum_{i_{c} \in I_{c}}\left(C_{i_{c}}^{+} p_{i_{c}, \omega, t}^{+}-C_{i_{c}}^{-} p_{i_{c}, \omega, t}^{-}\right) \\
& \left.\left.+C^{\mathrm{sh}, \mathrm{E}} l_{\omega, t}^{\mathrm{sh}, \mathrm{E}}+C^{\mathrm{sh}, \mathrm{G}} l_{\omega, t}^{\mathrm{sh}, \mathrm{G}}+\sum_{j \in J} C^{\mathrm{sp}} w_{j, \omega, t}^{\mathrm{sp}}\right)\right]
\end{aligned}
$$

subject to

$$
\begin{aligned}
& \text { constraints }(1 \mathrm{~b}),(1 \mathrm{~d})-(1 \mathrm{~h}), \\
& 0 \leq w_{j, t} \leq \bar{W}_{j}: \underline{\mu}_{j, t}^{\overline{\mathrm{W}}}, \bar{\mu}_{j, t}^{\mathrm{W}}, \quad \forall j, t, \\
& \text { constraints }(2 \mathrm{~b})-(2 \mathrm{p}), \quad \forall \omega,
\end{aligned}
$$

where $\Theta^{\mathrm{SC}}=\left\{p_{i, t}, \forall i, t ; w_{j, t}, \forall j, t ; p_{i, \omega, t}^{+}, p_{i, \omega, t}^{-}, \forall i, \omega, t ; g_{k, t}\right.$, $\left.\forall k, t ; w_{j, \omega, t}^{\mathrm{sp}}, \forall j, \omega, t ; g_{k, \omega, t}^{+}, g_{k, \omega, t}^{-}, \forall k, \omega, t ; l_{\omega, t}^{\mathrm{sh}, \mathrm{E}}, l_{\omega, t}^{\mathrm{sh}, \mathrm{G}}, \forall \omega, t\right\}$ is the set of primal optimization variables. In this model, the temporal coordination of the two trading floors is achieved through the real-time constraints (3d) for all scenarios $\omega \in \Omega$. The day-ahead dispatch of wind power is restricted by the installed capacity, instead of the expected wind generation and day-ahead dispatch decisions are treated as variables.

\section{Price-Based Coupled Electricity and Natural Gas Model}

The proposed dispatch model $(P-B)$ that aims at minimizing the expected cost of the integrated energy system and defining the optimal natural gas price adjustment $x_{t}$ writes as follows,

$\underset{\Theta^{\mathrm{UL}}}{\operatorname{Min}}(3 \mathrm{a})$

subject to

$$
\begin{aligned}
& -X \leq x_{t} \leq X, \forall t, \\
& \sum_{t \in T} \sum_{i_{g} \in I_{g}} \phi_{i_{g}} p_{i_{g}, t} x_{t}=0 \text {, } \\
& \left(p_{i, t}, w_{j, t}\right) \in \arg \{ \\
& \underset{\Theta_{1}^{\mathrm{LL}}}{\operatorname{Min}} \sum_{t \in T}\left(\sum_{i_{c} \in I_{c}} C_{i_{c}} p_{i_{c}, t}+\sum_{i_{g} \in I_{g}} C_{i_{g}, t} p_{i_{g}, t}\right) \\
& \text { subject to } \\
& \text { constraints }(1 \mathrm{~b})-(1 \mathrm{f}) \text {, } \\
& \left.C_{i_{g}, t}=\left(\hat{\lambda}_{t}^{\mathrm{G}}+x_{t}\right) \phi_{i_{g}}, \quad \forall i_{g}, t\right\}, \\
& \left(p_{i, \omega, t}^{+}, p_{i, \omega, t}^{-}, w_{j, \omega, t}^{\mathrm{sp}}, l_{\omega, t}^{\mathrm{sh}, \mathrm{E}}\right) \in \arg \{ \\
& \underset{\Theta_{2}^{\mathrm{LL}}}{\operatorname{Min}} \sum_{t \in T}\left(\sum_{i_{c} \in I_{c}}\left(C_{i_{c}}^{+} p_{i_{c}, \omega, t}^{+}-C_{i_{c}}^{-} p_{i_{c}, \omega, t}^{-}\right)\right. \\
& +\sum_{i_{g} \in I_{g}}\left(C_{i_{g}, \omega, t}^{+} p_{i_{g}, \omega, t}^{+}-C_{i_{g}, \omega, t}^{-} p_{i_{g}, \omega, t}^{-}\right) \\
& \left.+C^{\mathrm{sh}, \mathrm{E}} l_{\omega, t}^{\mathrm{sh}, \mathrm{E}}+\sum_{j \in J} C^{\mathrm{sp}} w_{j, \omega, t}^{\mathrm{sp}}\right)
\end{aligned}
$$

subject to

constraints $(2 \mathrm{~b})-(2 \mathrm{j})$,

$C_{i_{g}, \omega, t}^{+}=\left(\tilde{\lambda}_{\omega, t}^{\mathrm{G}}+x_{t}\right) \phi_{i_{g}}, \quad \forall i_{g}, t$,

$\left.C_{i_{g}, \omega, t}^{-}=\left(\tilde{\lambda}_{\omega, t}^{\mathrm{G}}+x_{t}\right) \phi_{i_{g}}, \forall i_{g}, t\right\}, \forall \omega$,

$$
\left(g_{k, t}, \hat{\lambda}_{t}^{\mathrm{G}}\right) \in \arg \{
$$

$\underset{\Theta_{3}^{\mathrm{LL}}}{\operatorname{Min}} \sum_{t \in T} \sum_{k \in K} C_{k} g_{k, t}$

subject to

constraints $(1 g)-(1 h)\}$, 


$$
\begin{aligned}
& \left(g_{k, \omega, t}^{+}, g_{k, \omega, t}^{-}, l_{\omega, t}^{\mathrm{sh}, \mathrm{G}}, \tilde{\lambda}_{\omega, t}^{G}\right) \in \arg \{ \\
& \quad \underset{\Theta_{4}^{\mathrm{LL}}}{\operatorname{Min}} \sum_{t \in T}\left(\sum_{k \in K}\left(C_{k}^{+} g_{k, \omega, t}^{+}-C_{k}^{-} g_{k, \omega, t}^{-}\right)\right) \\
& \left.\quad+C^{\mathrm{sh}, \mathrm{G}} l_{\omega, t}^{\mathrm{sh}, \mathrm{G}}\right) \\
& \quad \text { subject to } \\
& \quad \text { constraints }(2 \mathrm{k})-(2 \mathrm{p})\}, \forall \omega,
\end{aligned}
$$

where $\Theta_{1}^{\mathrm{LL}}=\left\{p_{i, t}, \forall i, t ; w_{j, t}, \forall j, t\right\}, \Theta_{2}^{\mathrm{LL}}=\left\{p_{i, \omega, t}^{+}, p_{i, \omega, t}^{-}\right.$, $\left.\forall i, \omega, t ; l_{\omega, t}^{\mathrm{sh}, \mathrm{E}}, \forall \omega, t ; w_{j, \omega, t}^{\mathrm{sp}}, \forall j, \omega, t\right\}, \Theta_{3}^{\mathrm{LL}}=\left\{g_{k, t}, \forall k, t\right\}$ and $\Theta_{4}^{\mathrm{LL}}=\left\{g_{k, \omega, t}^{+}, g_{k, \omega, t}^{-}, \forall k, \omega, t ; l_{\omega, t}^{\mathrm{sh}, \mathrm{G}}, \forall \omega, t\right\}$ are the sets of primal optimization variables of the lower-level problems. Additionally, $\Theta^{\mathrm{UL}}=\left\{x_{t}, \forall t, \Theta_{1}^{\mathrm{LL}}, \Theta_{2}^{\mathrm{LL}}, \Theta_{3}^{\mathrm{LL}}, \Theta_{4}^{\mathrm{LL}}\right\}$ is the set of primal optimization variables of the upper-level problem. The upper-level problem minimizes the expected cost of operating the integrated energy system by deciding the optimal value of variable $x_{t}$. Additionally, the lower-level problems practically reproduce the sequential coupled electricity and natural gas market. The system operator has the ability to vary the price of natural gas within specified limits, defined by (4b), to achieve a cost-effective system operation. The upperlevel variable $x_{t}$ has an impact on the decisions of the lowerlevel problems as the day-ahead and up/down regulation offer prices of NGFPPs are affected by this value through (4f), (4i) and $(4 \mathrm{j})$. Moreover, the lower-level decision variables affect the total expected cost of the integrated system. Capturing this dependency, the proposed mechanism can reveal the true value of the NGFPPs' flexibility and yield a dispatch that reduces expected system cost while respecting the merit-order principle. Equation (4c) acts as a cost-neutrality constraint since it guarantees that this mechanism leaves no financial deficit or surplus to the system operator, i.e., the hourly dayahead payments or charges are counterbalanced throughout the day. Potential deficit or surplus at the balancing stage is expected to be fairly limited and can be addressed through proper regulation as for instance the capacity payments for power availability in real-time operation.

The bilevel problem (4) can be reformulated as a Mathematical Program with Equilibrium Constraints (MPEC) by replacing the linear, and thus convex, lower level problems by their Karush-Kuhn-Tucker (KKT) conditions. Then, it is transformed into a Mixed-Integer Linear Program (MILP) in order to deal with the bilinear terms that arise from the complementarity conditions. Constraint (4c) is linearized by using the KKT conditions and strong duality theorem. A detailed mathematical description of the aforementioned procedure is presented in the electronic companion of the paper [11].

\section{RESULTS}

In this section, we illustrate the features of the three dispatch models presented in Section II. We consider a system that comprises three thermal power plants and two NGFPPs which also participate in the natural gas market to acquire their fuel. The unit data are provided in Table I. Up- and down-regulation offer prices are equal to 1.1 and 0.9 of day-ahead offer price.
The natural gas price adjustment is limited to $\$ 80 / \mathrm{kNm}^{3}$. The cost of load shedding is $\$ 1200 / \mathrm{MWh}$ and $\$ 1000 / \mathrm{kNm}^{3}$, while wind spillage is cost free. The peak natural gas and electricity demand are equal to $60 \mathrm{kNm}^{3} / \mathrm{h}$ and to $430 \mathrm{MW}$, respectively.

TABLE I

Electric Power and Natural Gas System Data

\begin{tabular}{cccccc|ccc}
\hline Unit $i$ & 1 & 2 & 3 & 4 & 5 & Unit $k$ & 1 & 2 \\
\hline$P_{i}^{\max }$ & 80 & 110 & 50 & 100 & 100 & $G_{k}^{\max }$ & 150 & 100 \\
$P_{i}^{+}$ & 10 & 0 & 30 & 25 & 20 & $G_{k}^{+}$ & 50 & 20 \\
$P_{i}^{-}$ & 10 & 0 & 30 & 25 & 20 & $G_{k}^{-}$ & 50 & 20 \\
$C_{i}$ & 30 & 10 & - & - & 60 & $C_{k}$ & 120 & 160 \\
$\phi_{i g}$ & - & - & 0.2 & 0.3 & - & & & \\
\hline
\end{tabular}

For illustration purposes, we perform a simulation in which wind power uncertainty is characterized by a set of two equiprobable scenarios, namely, $\omega_{1}(166 \mathrm{MW})$ and $\omega_{2}$ (86 MW). The marginal cost of wind power is equal to zero and the expected wind power production is $126 \mathrm{MW}$. We provide results for two time periods to show the effect of adjusted natural gas prices (either increased or reduced) on the schedules of NGFPPs. The results are presented in Tables II, III, IV and V. The lowest expected system cost is obtained by model Stoch. Model Seq respects the merit-order principle as wind power is dispatched to its expected value due to zero marginal cost and then the power plants are scheduled based on an ascending order of marginal costs. The marginal cost of NGFPPs stems from the multiplication of natural gas price and power conversion factor. In this example, natural gas is only produced by unit $K_{1}$, so its price is $\$ 120 / \mathrm{kNm}^{3}$, which results in a price offer from NGFPP $I_{4}$ equal to $\$ 36 / \mathrm{MWh}$. Unit $I_{5}$ is the most expensive and thus not scheduled at day-ahead stage.

Table II shows the generation schedule reported by models Seq and $P-B$, when the electricity demand is equal to $387 \mathrm{MW}$. In model $P-B$, variable $x_{t_{1}}$ is equal to $-\$ 20 / \mathrm{kNm}^{3}$ that results in a reduced natural gas price of $\$ 100 / \mathrm{kNm}^{3}$ for all NGFPPs, which in turn affects their marginal cost of power production. The marginal cost of NGFPP $I_{4}$ is reduced to $\$ 30 / \mathrm{MWh}$, which is equal to the marginal cost of unit $I_{1}$. NGFPP $I_{4}$ is now scheduled to $31 \mathrm{MW}$, while unit $I_{1}$ to $70 \mathrm{MW}$. The day-ahead cost increases compared to Seq since in reality the operating cost of the system rises from the actual costs and not the reduced one that NGFPPs buy natural gas. However, this increase is offset by the lower total balancing cost achieved by $P-B$. The up-regulation cost is lower since unit $I_{1}$ is able to provide a portion of the total $40 \mathrm{MW}$ needed for up-regulation. This is due to the fact that unit $I_{1}$ has a lower real upregulation cost than unit $I_{5}$. Moreover, NGFPP $I_{4}$ is more costeffective for providing down-regulation compared to NGFPP $I_{3}$ and thus reduces the down-regulation cost of $P-B$.

Similarly, Table IV shows the schedule of power plants under $S e q$ and $P-B$, when the electricity demand is equal to $344 \mathrm{MW}$. In this case, the value of $x_{t_{2}}$ is equal to $+\$ 30 / \mathrm{kNm}^{3}$ which in a similar manner affects the marginal cost of NGFPPs. The marginal cost of NGFPP $I_{3}$ is increased to $\$ 30 / \mathrm{MWh}$, which is equal to the marginal cost of unit $I_{1}$. This action results in an improved scheduling at the day-ahead stage that will result in a lower expected cost. In both cases, it can 
TABLE II

ELECTRIC POWER SYSTEM SCHEDULE IN MW $-\left(D^{\mathrm{E}}=387 \mathrm{MW}\right)$

\begin{tabular}{|c|c|c|c|c|c|c|}
\hline \multirow{3}{*}{ Agent } & \multicolumn{3}{|c|}{ Seq } & \multicolumn{3}{|c|}{ P-B } \\
\hline & \multirow[t]{2}{*}{ Day-ahead } & \multicolumn{2}{|c|}{ Balancing } & \multirow[t]{2}{*}{ Day-ahead } & \multicolumn{2}{|c|}{ Balancing } \\
\hline & & $\omega_{1}$ & $\omega_{2}$ & & $\omega_{1}$ & $\omega_{2}$ \\
\hline$I_{1}$ & 80 & -10 & 0 & 70 & -10 & +10 \\
\hline$I_{2}$ & 110 & 0 & 0 & 110 & 0 & 0 \\
\hline$I_{3}$ & 50 & -9 & 0 & 50 & -5 & 0 \\
\hline$I_{4}$ & 21 & -21 & +25 & 31 & -25 & +25 \\
\hline$I_{5}$ & 0 & 0 & +15 & 0 & 0 & +5 \\
\hline WP & 126 & +40 & -40 & 126 & +40 & -40 \\
\hline
\end{tabular}

TABLE III

EXPECTED SYSTEM OPERATION COST IN $\$-\left(D^{\mathrm{E}}=387 \mathrm{MW}\right)$

\begin{tabular}{cccccc}
\hline & Total & Day-ahead & Balancing & Up regulation & Down regulation \\
\hline Seq & 10400.4 & 9982.8 & 417.6 & 990.0 & -572.4 \\
Stoch & 10234.8 & 10222.8 & 12.0 & 660.0 & -648.0 \\
P-B & 10273.8 & 10042.8 & 231.0 & 825.0 & -594.0 \\
\hline
\end{tabular}

be observed that $x_{t}$ affects the natural gas price for power production, which in turn changes the day-ahead dispatch. The dispatch is changed in order to enable more cost-effective power plants to provide the required regulation. These price signals establish a temporal coordination between the two trading floors and thus better exploit the available technical flexibility of the two systems.

TABLE IV

ELECTRIC POWER SYSTEM SCHEDULE IN MW $-\left(D^{\mathrm{E}}=344 \mathrm{MW}\right)$

\begin{tabular}{cccc|cccc}
\hline \multirow{2}{*}{ Agent } & \multicolumn{3}{c|}{ Seq } & \multicolumn{3}{c}{ P-B } \\
\cline { 2 - 4 } \cline { 3 - 4 } & Day-ahead & \multicolumn{2}{c|}{ Balancing } & Day-ahead & \multicolumn{2}{c}{ Balancing } \\
\cline { 2 - 4 } \cline { 3 - 4 } & & $\omega_{1}$ & $\omega_{2}$ & & 70 & $\omega_{1}$ & $\omega_{2}$ \\
\hline$I_{1}$ & 58 & -10 & +10 & 110 & 0 & 0 \\
$I_{2}$ & 110 & 0 & 0 & 38 & -30 & +12 \\
$I_{3}$ & 50 & -30 & 0 & 0 & 0 & +18 \\
$I_{4}$ & 0 & 0 & +25 & 0 & 0 & 0 \\
$I_{5}$ & 0 & 0 & +5 & 0 & 0 \\
WP & 126 & +40 & -40 & 126 & +40 & -40 \\
\hline
\end{tabular}

TABLE V

EXPECTED SYSTEM OPERATION COST IN $\$-\left(D^{\mathrm{E}}=344 \mathrm{MW}\right)$

\begin{tabular}{cccccc}
\hline & Total & Day-ahead & Balancing & Up regulation & Down regulation \\
\hline Seq & 8932.8 & 8566.8 & 366.0 & 825.0 & -459.0 \\
Stoch & 8859.6 & 8206.8 & 652.8 & 917.4 & -264.6 \\
P-B & 8859.6 & 8638.8 & 220.8 & 679.8 & -459.0 \\
\hline
\end{tabular}

The following results are provided for the whole 24-hour scheduling horizon and 20 wind power scenarios (available at [12]). Fig. 1 shows the expected cost of the coupled electricity and natural gas system for different wind power penetration levels, i.e., share of installed wind power capacity on system's demand. It is observed that Stoch results in the lowest expected cost in all cases and efficiently utilizes the increase of wind power production. The expected cost of Seq diverges from the corresponding values of the other dispatch models for a wind power penetration level above $25 \%$ and shows a significant increase when this share is higher than $40 \%$. On the contrary, the expected cost of the proposed dispatch model $P-B$ remains close to Stoch over the whole range of wind power penetration. This verifies the ability of $P-B$ to bridge the gap between $S e q$ and Stoch by providing solutions closer to the stochastic ideal, while maintaining the economic properties of the sequential market clearing.

Additionally, Table VI presents the expected payment/charge to adjust the price of natural gas at the balancing stage and the overall savings in expected cost between Seq

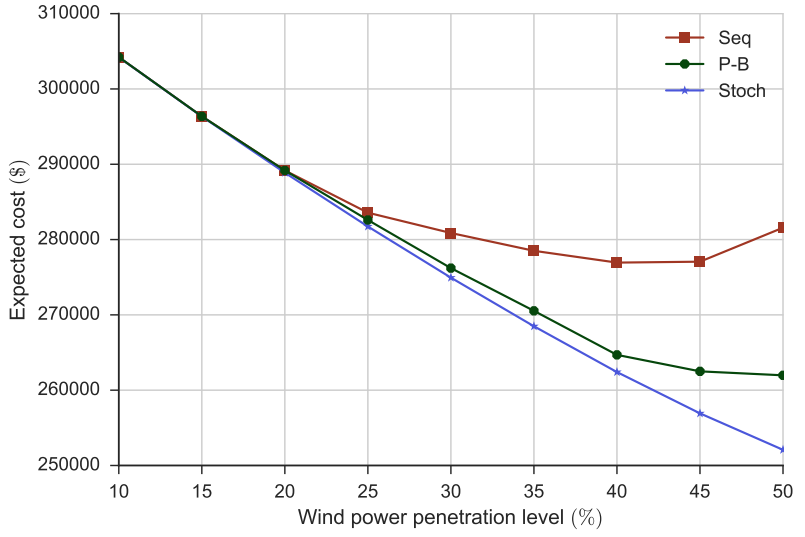

Fig. 1. Impact of wind power penetration level on the expected system cost.

TABLE VI

EXPECTED PAYMENT/CHARGE TO GENERATE FLEXIBILITY PRICE SIGNAL

\begin{tabular}{ccccccc}
\hline $\begin{array}{c}\text { Wind power } \\
\text { penetration level (\%) }\end{array}$ & 25 & 30 & 35 & 40 & 45 & 50 \\
\hline Exp. savings (\$) & 971.5 & 4663 & 8035.6 & 12251.8 & 14562.4 & 19601.6 \\
Exp. payment/charge (\$) & -352.1 & -177.1 & -21.4 & 133.5 & 2.2 & 302.3 \\
\hline
\end{tabular}

and $P-B$. Relevant results are only illustrated for wind power penetration levels that models $S e q$ and $P$ - $B$ provide a different dispatch. The payment/charge at the day-ahead stage is zero due to $(4 \mathrm{c})$ but at the balancing stage the system operator could have either a deficit or a surplus under different conditions. However, this financial imbalance is significantly lower than the benefit of reducing the total expected cost and can be either socialized or utilized for future investments. We observe larger expected savings as wind power penetration increases, while the expected payment/charge remains at the same level that is relatively small. For the case of $50 \%$ wind power penetration, $P-B$ reduces the expected cost by $\$ 19601.6$ compared to $\mathrm{Seq}$ and the system operator is expected to receive $\$ 302.3$.

We now consider a wind power penetration level of $50 \%$ to provide useful insights of the proposed dispatch model. Fig. 2 shows the natural gas price adjustment $\left(x_{t}\right)$ and the day-ahead payment/charge in order to generate this signal. It can be observed that the sign of $x_{t}$ determines whether the system operator has to incur a deficit or a surplus to change the price of natural gas, while the total settlement also depends on the volume of natural gas consumed for power production at the day-ahead stage. The natural gas price is reduced for the majority of time periods, which results in a deficit for the system operator during these hours. However, this deficit is offset by the surplus generated in periods when the natural gas price adjustment is positive, retaining this action as costneutral at the day-ahead stage.

Furthermore, we illustrate the natural gas price adjustment $\left(x_{t}\right)$ in relation to the difference in the hourly NGFPPs' share of the total power production between $P-B$ and Seq. A positive value (green area) shows that NGFPPs are scheduled to produce more at the day-ahead stage under $P-B$ than in $S e q$, while a negative value (red area) shows the opposite case. During the first hours of the day, inflexible unit $I_{2}$ is mainly dispatched under Seq. It can be observed that during the same 


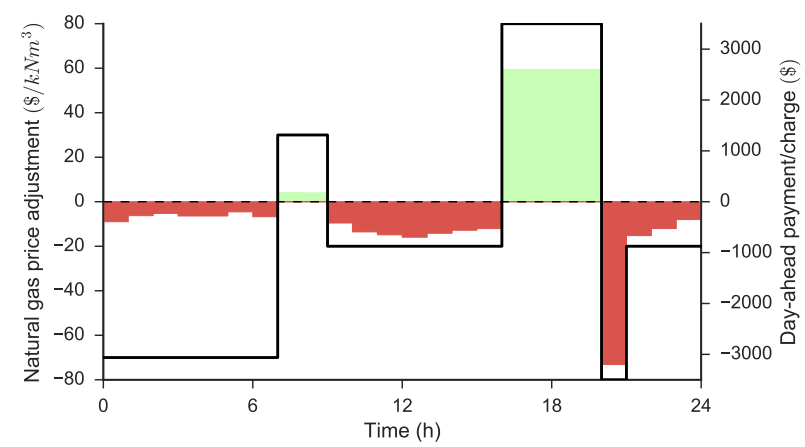

Fig. 2. Hourly natural gas price adjustment (black line: left y-axis) and dayahead financial settlement of the system operator to adjust the natural gas price (colored areas: right y-axis). Wind power penetration 50\%.

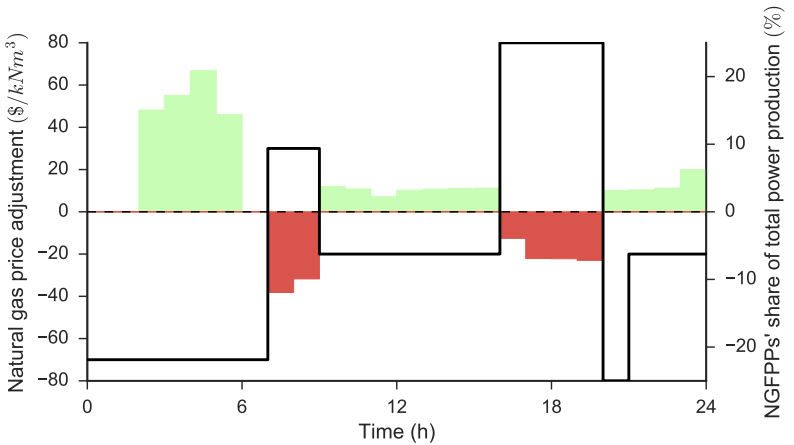

Fig. 3. Hourly natural gas price adjustment (black line: left y-axis) and difference in NGFPPs' share of total power production between $P-B$ and $S e q$ (colored areas: right y-axis). Wind power penetration $50 \%$.

period, the price of natural gas is reduced under $P-B$ in order to schedule NGFPP $I_{3}$ and exploit its flexibility. On the contrary, the price of natural gas is increased during hours 17-20, when electricity demand reaches its peak. As a result, a part of the electricity demand covered by NGFPP $I_{4}$ is undertaken by unit $I_{5}$ and that results in revealing flexibility to handle wind power uncertainty. Moreover, it is noticed that a change in the natural gas price does not always reflect an alternation of the day-ahead dispatch. This decision takes into account the tradeoff between improving the dispatch and guaranteeing that this action is cost-neutral at the day-ahead stage.

The pricing scheme of Stoch ensures cost recovery for flexible producers only in expectation. However, maintaining a sequential setup to clear the market generates prices that support the dispatch in such a way that the aforementioned property will hold for each scenario of stochastic production. We refer the reader to [8] for further discussion on this topic.

Supplementary results and the code are given in [11] for reader's convenience. The optimization problems were solved using CPLEX 12.6.2 under GAMS on a stationary computer with Inter i7 4-core processor clocking at $3.4 \mathrm{GHz}$ and $8 \mathrm{~GB}$ of RAM. The average time to solve $P-B$ was 520 seconds.

\section{CONCLUSION}

This paper proposes a price-based coordination between electricity and natural gas markets to bring the expected cost closer to the stochastic ideal solution. Using a natural gas price adjustment component, this mechanism enables an implicit temporal coupling of the day-ahead and balancing markets, while preserving the existing sequential market clearing of those trading floors. We employ a stochastic bilevel model that allows the system operator to anticipate the real-time operation of the integrated system taking into account the economic link between electricity and natural gas markets. The proposed method ensures that the natural gas price adjustment only affects the payment/charge at the balancing stage, where the traded quantities are significantly lower.

For future research, we intend to enrich the current model formulation including electricity network constraints. This will enable us to define more accurately the optimal natural gas price adjustments taking into account potential transmission congestions. The proposed model can be adapted to alternative coordination mechanisms between electricity and natural gas markets, e.g., quantity-based coordination as well as to compare the efficiency of these approaches against direct remuneration mechanisms of flexible producers.

\section{ACKNOWLEDGMENT}

The work of the authors is partly funded by the Danish Strategic Research Council (DSF) through projects 5s-Future Electricity Markets, No. 12-132636/DSF and CITIES, No. 1305-00027B/DSF. The authors would like to thank Tue V. Jensen for his help on supplementary material dissemination.

\section{REFERENCES}

[1] A. Zlotnik, L. Roald, S. Backhaus, M. Chertkov and G. Andersson, "Coordinated scheduling for interdependent electric power and natural gas infrastructures," IEEE Trans. on Power Syst., vol. 32, no. 1, pp. 600-610, 2017.

[2] C. M. Correa-Posada and P. Sánchez-Martin, "Integrated power and natural gas model for energy adequacy in short-term operation," IEEE Trans. on Power Syst., vol. 30, no. 6, pp. 3347-3355, 2015.

[3] B. Zhao, A. J. Conejo, and R. Sioshansi, "Unit commitment under gassupply uncertainty and gas-price variability," IEEE Trans. on Power Syst., doi:10.1109/TPWRS.2016.2602659.

[4] J. Gil, A. Caballero and A. J. Conejo, "Power cycling: CCGTs: The critical link between the electricity and natural gas markets," IEEE Power and Energy Magazine, vol. 12, no. 6, pp. 40-48, 2014.

[5] P. J. Hibbard and T. Schatzki, "The interdependence of electricity and natural gas: Current factors and future prospects," Electricity J., vol. 25 , no. 4, pp. 6-17, 2012.

[6] P. Pinson, L. Mitridati, C. Ordoudis and J. Østergaard, "Towards fully renewable energy systems - Experiences and trends in Denmark," CSEE Journal of Power and Energy Systems, 2016, in press.

[7] G. Pritchard, G. Zakeri and A. Philpott, "A single-settlement, energyonly electric power market for unpredictable and intermittent participants," Oper. Res., vol. 58, no. 4, pp. 1210-1219, 2010.

[8] J. M. Morales, M. Zugno, S. Pineda and P. Pinson, "Electricity market clearing with improved scheduling of stochastic production," Eur. J. Oper. Res., vol. 235, no. 3, pp. 765-774, 2014.

[9] A. Alabdulwahab, A. Abusorrah, X. Zhang and M. Shahidehpour, "Coordination of interdependent natural gas and electricity infrastructures for firming the variability of wind energy in stochastic day-ahead scheduling," IEEE Trans. Sustain. Energy, vol. 6, no. 2, pp. 606-615, 2015.

[10] A. Henriot and J. M. Glachant, "Capacity remuneration mechanisms in the European market: now but how?," European University Institute (EUI), Robert Schuman Centre of Advanced Studies (RSCAS), 2014.

[11] C. Ordoudis, S. Delikaraoglou, P. Pinson and J. Kazempour, "Electronic companion for paper: Exploiting flexibility in coupled electricity and natural gas markets: A price-based approach", [Online], http://doi.org/10.5281/zenodo.376307.

[12] W. Bukhsh, "Data for stochastic multiperiod optimal power flow problem," Website, Mar. 2017, https://sites.google.com/site/datasmopf/. 